\title{
Hash-Based Structural Join Algorithms
}

\author{
Christian Mathis and Theo Härder \\ University of Kaiserslautern ${ }^{\star \star}$
}

\begin{abstract}
Algorithms for processing Structural Joins embody essential building blocks for XML query evaluation. Their design is a difficult task, because they have to satisfy many requirements, e. g., guarantee linear worst-case runtime; generate sorted, duplicate-free output; adapt to fiercely varying input sizes and element distributions; enable pipelining; and (probably) more. Therefore, it is not possible to design the structural join algorithm. Rather, the provision of different specialized operators, from which the query optimizer can choose, is beneficial for query efficiency. We propose new hash-based structural joins that can process unordered input sequences possibly containing duplicates. We also show that these algorithms can substantially reduce the number of sort operations on intermediate results for (complex) tree structured queries (twigs).
\end{abstract}

\section{Introduction}

Because XML data is based on a tree-structured data model, it is natural to use path and tree patterns for the search of structurally related XML elements. Therefore, expressions specifying those patterns are a common and frequently used idiom in many XML query languages and their effective evaluation is of utmost importance for every XML query processor. A particular path pattern — the twig - has gained much attention in recent publications, because it represents a small but frequently used class of queries, for which effective evaluation algorithms have been found $[1,3,7,11,14,16]$.

Basically, a twig, as depicted in Fig. 1, is a small tree, whose nodes $n$ represent simple predicates $p_{n}$ on the content (text) or the structure (elements) of an XML document, whereas its edges define the relationship between the items to match. In the graphical notation, we use the double line for the descendant and the single line for the child relationship. For twig query matching, the query processor has to find all possible embeddings of the given twig in the queried document, such

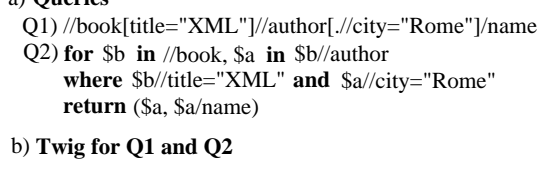

Fig. 1. Sample Query and Twig that each node corresponds to an XML item and the defined relationship among the matched items is fulfilled. The result of a twig is represented as an ordered ${ }^{1}$ sequence $^{-}$

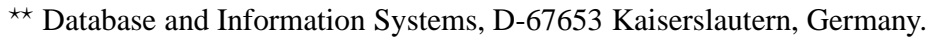

\{mathis|haerder\}@informatik.uni-kl.de

1 Here, "ordered" means: sorted in document order from the root to the leaf items.
} 
of tuples, where the fields of each tuple correspond to matched items. Usually, not all nodes of a twig generate output, but are mere (path) predicates. Therefore, we use the term extraction point [7] to denote twig nodes that do generate output (the boxed nodes in Fig. 1).

\subsection{Related Work}

For twig query matching, a large class of effective methods builds on two basic ideas: the structural join [1] and the holistic twig join [3]. The first approach decomposes the twig into a set of binary join operations, each applied to neighbor nodes of the twig (for an example, see Fig. 2). The result of a single join operation is a sequence of tuples $S_{\text {out }}$ whose degree (number of fields) is equal to the sum of the degrees of its input tuples from sequences $S_{i n A}$ and $S_{i n B}$. $S_{\text {out }}$ may serve as an input sequence for further join operations. In the following, we denote the tuple fields that correspond to the twig nodes to join as the join fields. The underlying structural join algorithms are interchangeable and subject to current research (see the discussion below).

In [3], the authors argue that, intrinsic for the structural join approach, intermediate result sizes may get very large, even if the final result is small, because the intermediate result has to be unnested. In the worst case, the size of an intermediate result sequence is in the order of the product of the sizes of the input sequences. To remedy this drawback, twig join algorithms $[3,7]$ evaluate the twig as a whole, avoiding intermediate result unnesting by encoding the qualifying elements on a set of stacks.

Of course, holistic twig join algorithms are good candidates for physical operators supporting query evaluation in XDBMSs. However, they only provide for a small fraction of the functionality required by complete XPath and XQuery processors (e. g., no processing of axes other than child and descendant; no processing of order-based queries). Therefore, the development of new structural join algorithms is still valuable, because they can act as complemental operators in case the restricted functionality of twig joins is too small, or as alternatives if they promise faster query evaluation.

Existing structural join approaches can roughly be divided into four classes by the requirements they pose on their input sequences: A) no requirements $[8,11,14]$; B) indexed input [16], C) sorted input [1,10, 16]; D) indexed and sorted input [4]. Especially for classes $\mathrm{C}$ and $\mathrm{D}$, efficient algorithms have been found that generate results in linear time depending on the size of their input lists. In contrast, for class A, there is- to the best of our knowledge - no such algorithm. All proposed approaches either sort at least one input sequence [11], or create an in-memory data structure (a heap) requiring $O\left(n \log _{2} n\right)$ processing steps [14]. By utilizing hash tables that can be built and probed in (nearly) linear time, the algorithms we introduce in this paper can remedy this problem. Note, the strategies in $[11,14]$ elaborate on partition-based processing schemes, i. e., they assume a small amount of main memory and large input sequences, requiring their partition-wise processing. Their core join algorithm, however, is main-memorybased, as ours is. Therefore, our new join operators can be-at least theoretically ${ }^{2}-$ combined with the partitioning schemes proposed in these earlier works. Answering

\footnotetext{
2 [14] uses a perfect binary tree (PBiTree) to generate XML identifiers. In real-world scenarios, we assume document modifications that can hardly be handled with PBiTrees. Therefore, we
} 
twig (and more complex queries) using binary structural join algorithms imposes three non-trivial problems: selecting the best (cheapest) join order $(P I)$ to produce a sorted $(P 2)$ and duplicate-free $(P 3)$ output. P1 is tackled in [15], where a dynamic programming framework is presented that produces query executions plans (QEPs) based on cost estimations. The authors assume class C (and D) algorithms, which means that even intermediate results are required to be in document order on the two join fields. As a consequence, sort operators have to be embedded into a plan to fulfill this requirement. Consider for example the twig in Fig. 1. Let the circled numbers denote the join order selected by an algorithm from [15]. Then, three sort operators have to be embedded into the QEP ( $\mathrm{see}^{3}$ Fig. 2). Sort operators are expensive and should be avoided whenever possible. With structural join algorithms not relying on a special input order-like those presented in this paper-we can simply omit the sort operators in this plan. However, a final sort may still be necessary in some cases.

Problem P3 was studied in [8]. The authors show that duplicate removal is also important for intermediate results, because otherwise, the complexity of query evaluation depending on the number of joins for a query $\mathrm{Q}$ can lead to an exponential worst-case runtime behavior. Therefore, for query evaluation using binary structural joins, tuplewise duplicate-free intermediate result sequences have to be assured after each join execution. Note, due to result unnesting, even a (single) field in the tuple may contain duplicates. This

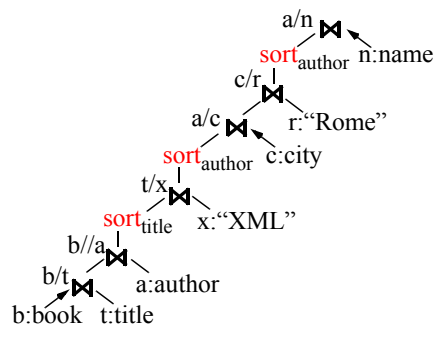

Fig. 2. Sample Plan circumstance is unavoidable and, thus, we have to cope with it. Because duplicate removal - like the sort operator - is an expensive operation, it should be minimized. For example in [6], the authors present an automaton that rewrites a QEP for Q, thereby removing unnecessary sort and duplicate removal operations. Their strategy is based on plans generated by normalization of XPath expressions, resulting in the XPath core language expressions. However, this approach does not take join reordering into account, as we do. Our solution to P3 is a class of algorithms that do not produce any duplicates if their input is duplicate free.

\subsection{Contribution}

We explore the use of hash-based joins for path processing steps of XML queries and identify the selectivity ranges when they are beneficial. In particular, we propose a class of hash-based binary structural join operators for the axes parent, child, ancestor, descendant, preceding-sibling, and following-sibling that process unordered input sequences and produce (unordered) duplicate-free output sequences. Furthermore, we show by extensive tests using the XTC (XML Transaction Coordinator)-our prototype of a native XDBMS - that our approach leads to a better runtime performance than sort-based schemes.

The remainder of this paper is organized as follows: Sect. 2 briefly describes some important internals of XTC, namely our node labeling scheme and an access method for

used SPLIDs (Sect. 2.1) instead. As a consequence, this "gap" had to be bridged to support the proposed partition schemes with our ideas.

${ }^{3}$ An arrow declares the input node of a join by which the output is ordered, where important. Possible are root to leaf, e. g., between "book" and "title", and leaf to root, e. g., the final join. 
element sequences. Sect. 3 introduces new hash-based algorithms. In Sect. 4 we present our quantitative results before we conclude in Sect. 5 .

\section{System Testbed}

XTC adheres to the well-known layered hierarchical architecture: The concepts of the storage system and buffer management could be adopted from existing relational DBMSs. The access system, however, required new concepts for document storage, indexing, and modification including locking. The data system available only in a slim version is of minor importance for our considerations.

\subsection{Path Labels}

Our comparison and evaluation of node labeling schemes in [9] recommends node labeling schemes which are based on the Dewey Decimal Classification [5]. The abstract properties of Dewey order encoding - each label represents the path from the documents root to the node and the local order w.r.t. the parent node; in addition, sparse numbering facilitates node insertions and deletions_-are described in [13]. Refining this idea, similar labeling schemes were proposed which differ in some aspects such as overflow technique for dynamically inserted nodes, attribute node labeling, or encoding mechanism. Examples of these schemes are ORDPATH [12], DeweyID [9], or DLN [2]. Because all of them are adequate and equivalent for our processing tasks, we prefer to use the substitutional name stable path labeling identifiers (SPLIDs) for them.

Here we only summarize the benefits of the SPLID concept which provides holistic system support. Existing SPLIDs are immutable, that is, they allow the assignment of new IDs without the need to reorganize the IDs of nodes present. Comparison of two SPLIDs allows ordering of the respective nodes in document order, as well as the decision of all XPath axis relations. As opposed to competing schemes, SPLIDs easily provide the IDs of all ancestors to enable direct parent/ancestor identification or access. This property is very helpful for navigation and for fine-grained lock management in the XML documents. Finally, the representation of SPLIDs, e. g., label 1.3.7 for a node at level 3 and also used as an index reference to this node, facilitates the application of hashing in our join algorithms.

\subsection{Accessing Ordered Element Sequences}

A B*-tree is used as a document store where the SPLIDs in inner B*-tree nodes serve as fingerposts to the leaf pages. The set of doubly chained leaf pages forms the so-called document container where the XML tree nodes are stored using the format (SPLID, data) in document order. Important for our discussion, the XDBMS

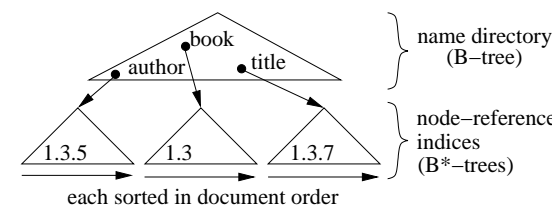

Fig. 3. Element Index 
creates an element index for each XML document. This index consists of a name directory with (potentially) all element names occurring in the XML document (Fig. 3). For each specific element name, in turn, a node-reference index is maintained which addresses the corresponding elements using their SPLIDs. Note, for the document store and the element index, prefix compression of SPLID keys is very effective because both are organized in document order directly reflected by the SPLIDs [9].

The leaf nodes in our QEPs are either element names or values. By accessing the corresponding node reference indexes, we obtain for them ordered lists of SPLIDs and, if required lists of nodes in document order by accessing the document store.

\section{Hash-Based Structural Join Algorithms}

To be able to compete with existing structural join algorithms, we had to design our new algorithms with special care. In particular, the use of semi-joins has several important benefits. The processing algorithms become simpler and the intermediate result size is reduced (because the absolute byte size is smaller and we avoid unnesting). Several important design objectives can be pointed out:

Design single-pass algorithms. As in almost all

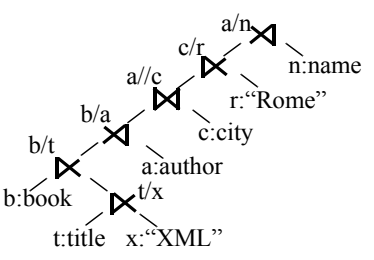

Fig. 4. Plan for Query 1 other structural join proposals, we have to avoid multiple scans over input sequences.

Exploit extraction points. With knowledge about extraction points, the query optimizer can pick semi-join algorithms instead of full joins for the generation of a QEP. For example, consider the plan in Fig. 4 which embodies one way to evaluate the twig for the XPath expression in Fig. 1. After having joined the title elements with the content elements "XML", the latter ones are not needed anymore for the evaluation of the rest of the query; a semi-join suffices.

Enable join reordering. Join reordering is crucial for the query optimizer which should be able to plan the query evaluation with any join order to exploit given data distributions. As a consequence, we need operators for the reverse axes ancestor and parent, too (e. g., the semi-join operator between title and "XML" in Fig. 4 actually calculates the parent axis).

Avoid duplicate removal and sort operations whenever possible. By using only algorithms that do not generate duplicates and operate on unordered input sequences, the query optimizer can ignore these problems. However, the optimizer has to ensure the correct output order, requiring a final sort operator. In some cases, this operator can be skipped: If we assume that the element scans at the leaf nodes of the operator tree in Fig. 4 return the queried element sequences in document order (as, for example, our element index assures), then, because the last semi-join operator is simply a filter for name elements (see Sect. 3.1), the correct output order is automatically established.

Design dual algorithms that can hash the smaller input sequence. The construction of an in-memory hash table is still an expensive operation. Therefore, our set of algorithms should enable the query optimizer to pick an operator that hashes the smaller of both input sequences and probes the other one, yielding the same result. 
Table 1. Classification of Hash-Join Operators

\begin{tabular}{|c|c|c|c|}
\hline Hashed & ancestor/parent & $\begin{array}{c}\text { Output } \\
\text { |descendant/child }\end{array}$ & full join \\
\hline $\begin{array}{l}\text { parent } \\
\text { ancestor }\end{array}$ & $\begin{array}{l}\text { Class 1: UpStep } \\
/ / \mathbf{a}[\mathrm{b}] \\
\text { ParHashA } \\
/ / \mathbf{a}[\cdot / / \mathrm{b}] \\
\text { AncHashA } \\
\end{array}$ & $\begin{array}{l}\text { Class 2: TopFilter } \\
\text { //a/b } \\
\text { ChildHashA } \\
\text { //a//b } \\
\text { DescHashA }\end{array}$ & $\begin{array}{l}\text { Class 3: FullTopJoin } \\
/ / \mathbf{a} / \mathrm{b}, / / \mathbf{a}[\mathrm{b}] \\
\text { ChildFullHashA } \\
/ / \mathbf{a} / / \mathrm{b}, / / \mathbf{a}[. / / \mathrm{b}] \\
\text { DescFullHashA }\end{array}$ \\
\hline $\begin{array}{l}\text { child } \\
\text { descendant }\end{array}$ & \begin{tabular}{|l} 
Class 4: BottomFilter \\
$/ /$ a $[\mathbf{b}]$ \\
ParHashB \\
$/ /$ a $[. / / \mathbf{b}]$ \\
AncHashB
\end{tabular} & $\begin{array}{l}\text { Class 5: DownStep } \\
\text { //a/b } \\
\text { ChildHashB } \\
\text { //a//b } \\
\text { DescHashB }\end{array}$ & $\begin{array}{l}\text { Class 6: FullBottomJoin } \\
\text { //a/b, //a [b] } \\
\text { ChildFullHashB } \\
\text { //a//b, //a }[/ / / b] \\
\text { DescFullHashB }\end{array}$ \\
\hline
\end{tabular}

\subsection{Classification of Algorithms}

We can infer three orthogonal degrees of freedom for structural hash-join algorithms: the axis that has to be evaluated (parent/child/ancestor/descendant); the mode of the join (semilfull); and the choice of which input sequence to hash $(A \text { or } B)^{4}$. The following naming scheme is used for our operators: $\langle$ axis $\rangle+\langle$ mode $\rangle+\langle$ hash $\rangle$ : $\{$ Par $\mid$ Child $\mid$ Anc $\mid$ Desc $\}\{\operatorname{Semi} \mid \operatorname{Full}\} \operatorname{Hash}\{A \mid B\}$ "Semi" is omitted for brevity). For example, the join operator between title and "XML" in Fig. 4 is a ParHashB operator, because it calculates the parent axis, is a semi-join operator, and hashes the sequence of possible children.

For an overview of all possible operators refer to Table 1: The column header defines the input to be hashed, whereas the row header defines the output. For clarification of the semantics, each operator is additionally described by an XPath expression where the input sequence to hash is marked in bold face. The names of the operator classes describe the evaluation strategy of the join. They will be discussed in the following. Note, class 1-3 algorithms are dual to class 4-6 algorithms, i. e., they calculate the same result as their corresponding algorithms, but hash a different input sequence.

\subsection{Implementation}

To abstract from operator scheduling and dataflow control, we let all operators act in the same operating system thread and use the well-known iterator-based open-next-close protocol as a basis for the evaluation. Each algorithm receives two input sequences of tuples, where, due to intermediate result unnesting, duplicates on the join fields have to be expected.

All proposed algorithms in this paper consist of two phases. In phase one, a hash table ht is constructed using the join field of the tuples of one input sequence (either sequence A or B). In phase 2, the join field of the other input sequence is probed against ht. Depending on how a result tuple is constructed, the operators can be assigned to one of the six classes: Full $*$ Join operators return a sequence of joined result tuples just as earlier proposals for structural join algorithms (e. g., [1]). Note, the qualifiers "Top" and "Bottom" denote which input sequence is hashed. The remaining classes contain

\footnotetext{
${ }^{4}$ Note, in the following, $A$ denotes the sequence of possible ancestors or parents (depending on the context), whereas $B$ denotes descendants or children.
} 


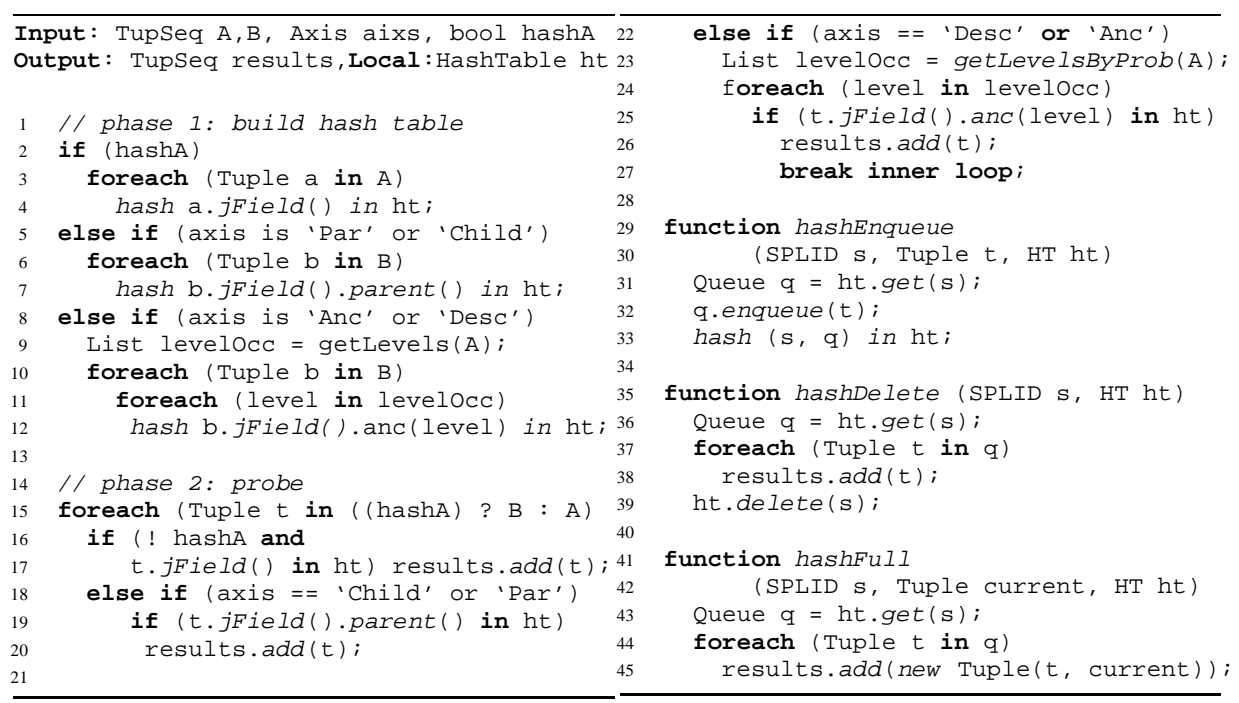

Fig. 5. *Filter Operator and Auxiliary Functions for *Step and Full*Join

semi-join algorithms. *Filter operators use the hash table, constructed for one input sequence to filter the other one, i. e., tuples are only returned from the probed sequence. *Step operators work the other way around, i.e., they construct the result tuples from the hashed input sequence.

*Filter Operators (see Fig. 5): In phase one, for ChildHashA and DescHashA, the algorithm simply hashes the SPLID of the elements of the join fields (accessed via method jField ()) into ht (line 4). Then, in phase two, the algorithm checks for each tuple $t$ in B, whether the parent SPLID (line 19 for ChildHashA) or any ancestor SPLID (line 25 for DescHashA) of the join field is contained in ht. If so, $t$ is a match and is appended to the result. Actually, for the descendant operator, we had to check all possible ancestor SPLIDs which could be very costly. To narrow down the search, we use the meta-information, at which levels and by which probability an element of the join field of A occurs (line 23). This information can be derived dynamically, e. g., when the corresponding elements are accessed via an element index scan, or kept statically in the document catalog.

The strategy for ParHashB and AncHashB is similar, with the difference, that in the hash phase the algorithm uses the join fields of input B to precalculate SPLIDs that might occur in A (lines 7 and 12). Again for the descendant operator, we use the level information (line 9), but this time the probability distribution does not matter. In the probing phase it only has to be checked, whether the current join field value is in ht.

Obviously, the output order of the result tuples is equal to the order of the probed input sequence. Furthermore, if the probed input sequence is tuplewise duplicate free, the algorithm does not produce any duplicates. The hashed input sequence may contain duplicates. However, these are automatically skipped, whereas collisions are internally resolved by the hash table implementation. 
*Step Operators conceptually work in the same way as their corresponding *Filter operators. However, they do not return tuples from the probed, but from the hashed input sequence. Accordingly, tuples that have duplicates on the join field (e. g., TupSeq A of Fig. 6a) may not be skipped (as above) but have to be memorized for later output. The new algorithms work as follows: In the hash phase, the function hashEnqueue () (Fig. 5 line 29) is called instead of the simple hash statements in lines 4, 7, and 12). The first argument is the SPLID $s$ of the join field (or its parentlancestor SPLID). Function hashEnqueue() checks for $s$ whether or not an entry is found in hash table ht (line 31 ). If so, the corresponding value, a queue $q$, is returned to which the current tuple is appended (line 32). Finally, $q$ is written back into the hash table (line 33).

In the probing phase, we substitute the hash table lookup and result generation (lines 17, 19-20, 25-26) with the hashDelete () method (Fig. 5 line 35). For the given SPLID s to probe, this method looks up the corresponding tuple queue in the hash table and adds each contained tuple $t$ to the result. Finally, the entry for $s$ and its queue are removed from the hash table, because the result tuples have to be returned exactly once to avoid duplicates. The sort order of these algorithms is dictated by the sort order of the input sequence used for probing. If the hashed input sequence did not contain any duplicates, the result is also duplicate free.

Full*Join Operators resemble the *Step operators. The only difference is the result generation. While *Step algorithms are semi-join operators that do not produce a joined result tuple, Full*Join operators append the current result tuple with all tuples matched (as depicted in method hashFull (), Fig. 5 line 41). Note, opposed to hashDelete (), in hashFull() no matched entries from ht are deleted. For a brief full join example see Fig.6a: input sequence A for the ChildFullHashA operator is hashed on join field 1 , thereby memorizing tuples with duplicates in the related queues. Then, the tuples from sequence B are probed against the hash table. For each match, each tuple in the queue is joined with the current tuple from B and appended to the result.

Space and Time Complexity. The space complexity (number of tuples stored) and time complexity (number of hashes computed) of the operators depend on the axis to be evaluated. Let $n=|A|$ and $m=|B|$ be the sizes of the input sequences. For the parent/child axis, the space and time complexity is $O(n+m)$. For the ancestor/descendant axis, the height $h$ of the document also plays a role. Here the space complexity for classes $1-3$ is also $O(n+m)$, whereas the time complexity is $O(n+h * m)$ (for each tuple in sequence B up to $h$ hashes have to be computed). For classes 4-6, both space and time complexity are $O(n+h * m)$.

Beyond Twig Functionality: Calculation of Sibling Axes. With hash-based schemes and a labeling mechanism enabling the parent identification, the preceding-sibling and the following-sibling axes are-in contrast to holistic twig join algorithms-computable, too. Due to space restrictions, we can only show filtering algorithms, corresponding to the *Filter classes above: In phase 1 operators PreSiblHashA and FollSiblHashA (see Fig. 6b) create a hash table ht to store key-value pairs of parent/child SPLIDs. For each element in A, parent $p$ is calculated. Then the following-sibling 

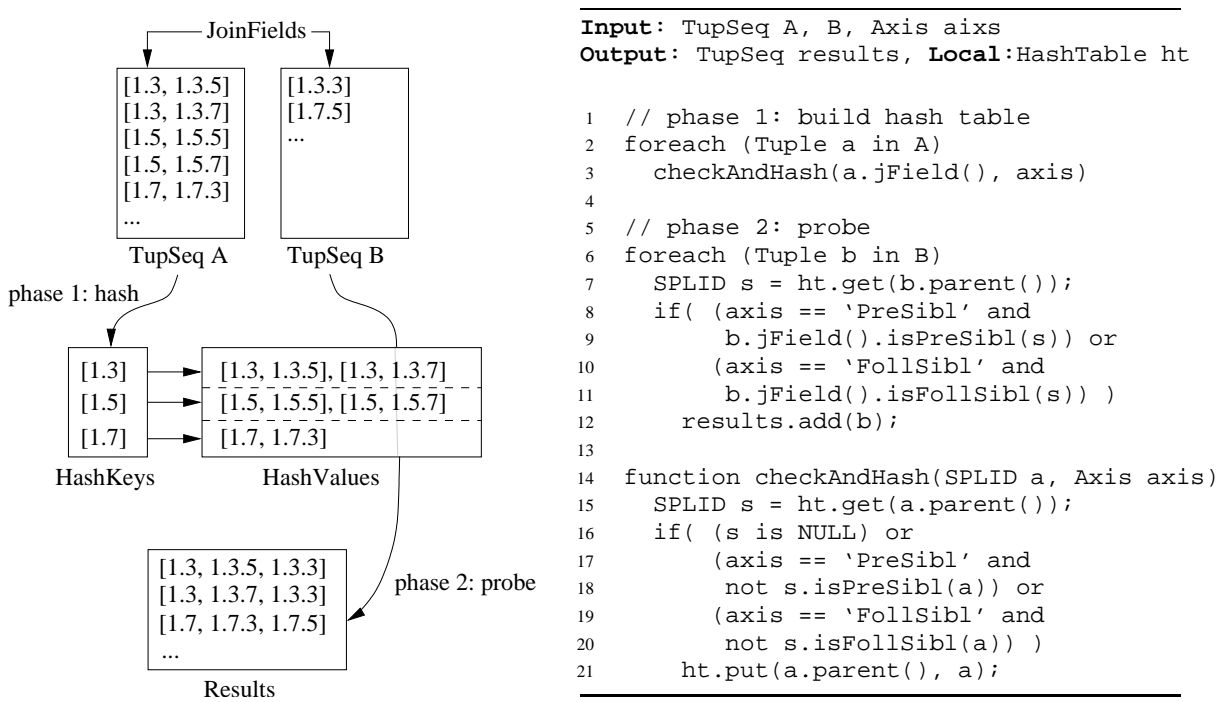

Fig. 6. a) Full*Join Example and b) Sibling Operator

(preceding-sibling) axis is evaluated as follows: For each parent SPLID $p$, the smallest (largest) child SPLID $c$ in A is stored in ht. This hash table instance is calculated by successive calls to the checkAndHash () method (lines 14 to 21). While probing a tuple $b$ of input $\mathrm{B}$, the algorithm checks whether the SPLID on the join field of $b$ is a following-sibling (preceding-sibling) of $c$, that has the same parent (lines 6 to 12). If so, the current $b$ tuple is added to the result. Clearly, these algorithms reveal the same characteristics as their corresponding *Filter algorithms: They do not produce any tuplewise duplicates and preserve the order of input sequence B.

\section{Quantitative Results}

To substantiate our findings, we compared the different algorithms by one-to-one operator comparison on a single-user system. All tests were run on an Intel XEON computer (four $1.5 \mathrm{GHz}$ CPUs, $2 \mathrm{~GB}$ main memory, $300 \mathrm{~GB}$ external memory, Java Sun JDK 1.5.0) as the XDBMS server machine and a PC (1.4 GHz Pentium IV CPU, $512 \mathrm{MB}$ main memory, JDK 1.5.0) as the client, connected via 100 MBit ethernet to the server.

To test the dependency between runtime performance and query selectivity, we generated a collection of synthetic XML documents, whose structure is sketched in Fig. 7. Each document has a size of $200 \mathrm{MB}$ and contains bibliographic information. Because we were mainly interested in structural join operators for element sequences, the generated documents do not contain much text content. The schema graph is a directed acyclic graph (and not a tree), because an author element may be the child of either a book or an article element. We generated the documents in such a way, that we obtained the following selectivity values for the execution of structural joins between input nodes: $1 \%, 5 \%, 10 \%, 50 \%$, and $100 \%$. For example, for the query //book [title], 

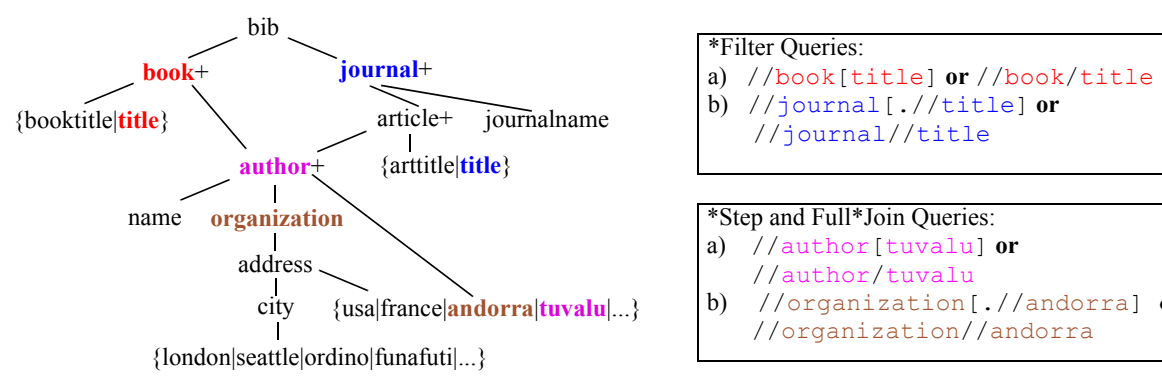

Fig. 7. Document Schema and Sample Queries

selectivity $1 \%$ means that $1 \%$ of all title elements have a book element as their parent (all others have the article element as parent). Additionally, we created 10\% noise on each input node, e. g., $10 \%$ of all book elements have the child booktitle instead of title.

\subsection{Join Selectivity Dependency of Hash-Based Operators}

In a first experiment, we want to explore the influence of the join selectivities of the input sequences and, in case of varying input sequence sizes, their sensitivity on the hash operator performance. All operators presented in Table 1 revealed the same performance characteristics as a function of the join selectivity. Hence, it is sufficient to present an indicative example for which we have chosen the DescFullHash * operators. For the query / / journal//title, the size of the input sequence containing journal elements varies from around 2,000 to 200,000 elements, whereas the size of the title sequence remains stable (roughly 200,000 elements). Fig. 8a illustrates the runtime performance of the DescFullHashA operator and the DescFullHashB operator for the same query. For selectivities smaller than $10 \%$, the runtime of each operator remains quite the same, because in these cases external memory access costs for the node reference indexes (column sockets) dominate the execution time, whereas the time for the hash table creation and probing remains roughly the same. However for selectivities $>$ $10 \%$, the runtime increases due to higher CPU costs for hashing and probing of larger input sequences. The gap between the DescFullHashA and the DescFull HashB operator results from hashing the wrong-i.e., the larger-input sequence (title) instead of the smaller one (in operator DescFull HashB). Therefore, it is important that the query optimizer chooses the right operator for an anticipated data distribution.

\subsection{Hash-Based vs. Sort-Based Schemes}

In the next test, we want to identify the performance differences of our hash-based schemes as compared to sort-based schemes. For this purpose, we implemented the StackTree algorithm [1] and the structural join strategy from [14] called AxisSort* in the following. Both operators work in two phases: In phase 1, input sequences are sorted using the QuickSort algorithm. While StackTree needs to sort both input sequences, 

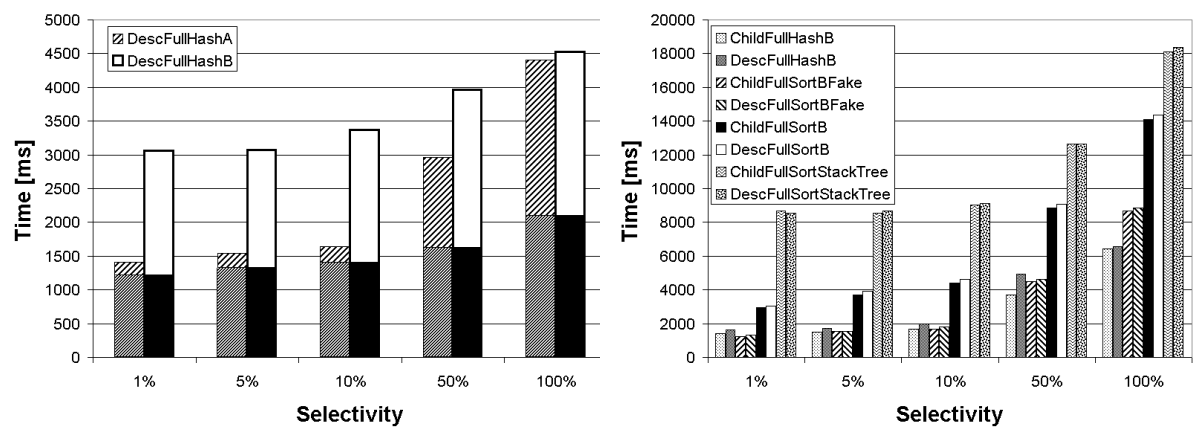

Fig. 8. a) DescFullHash* Characteristics, b) Operator Comparison

AxisSort* only needs to sort the smaller one. In phase 2, StackTree accomplishes its ordinary join strategy, while AxisSort* performs a binary search on the sorted input for each element of the other input sequence. To compare our operators with minimal-cost sort-based schemes, we introduce hypothetical operators which also sort the smaller input sequence, but omit the probing phase. Thus, so-called *Fake operators do not produce any output tuples. The result comparison is presented in Fig. 8b. Having the same join selectivity dependency, our hash-based operators are approximately twice as fast as the sort-based operators (with result construction). The figures for the StackTree algorithm impressively demonstrate that sort operations on intermediate results in query plans should really be avoided if possible. Finally, the hash-based operators-with their "handicap" to produce a result-match the sort-based fake operators.

\subsection{Memory Consumption}

Finally, we measured the memory consumption of hash-based and sort-based operators. On the generated document collection, we issued the query //organization [.//andorra], where the number of andorra elements varied from 2000 to 200.000, whereas organization elements remained stable (at roughly 200.000). For comparison, we used the DescFullHashB ${ }^{5}$ and the DescFullSortB operator. In all selectivity ranges, the internal hash table of the hash-based operator consumed three to four times more memory than the plain array of the sort-based one. To reduce this gap, a space optimization for hash-based operators is possible: Each key contained in the hash-table (as depicted in Fig. 6a) is repeated (as a prefix) in the join field value of the tuples contained in the key's queue. This redundant information can safely be disposed for a more compact hash table.

In a last experiment, we compare DescFull HashB with AncHashB. Here, the semi-join alternative required around three times fewer memory than the full join variant on all selectivities. This circumstance is also a strong argument for our proposal, that the query optimizer should pick semi-join operators whenever possible.

\footnotetext{
${ }^{5}$ Note, regarding the space complexity, DescFull HashB is one of the more expensive representative among the hash-based operators (see 3.2).
} 


\section{Conclusions}

In this paper, we have considered the improvement of twig pattern queries-a key requirement for XML query evaluation. For this purpose, we have substantially extended the work on structural join algorithms thereby focussing on hashing support. While processing twig patterns, our algorithms, supported by appropriate document store and index structures, primarily rely on SPLIDs which flexibly enable and improve path processing steps by introducing several new degrees of freedom when designing physical operators for path processing steps.

Performance measurements approved our expectations about hash-based operators. They are, in the selectivity range $1 \%-100 \%$, twice as fast as sort-based schemes and not slower than the *Fake operators. As another beneficial aspect, intermediate sorts in QEPs can be drastically reduced. Such hash-based operators should be providedpossibly with other kinds of index-based join operators - in a tool box for the cost-based query optimizer to provide for the best QEP generation in all situations.

\section{References}

1. S. Al-Khalifa et al.: Structural Joins: A Primitive for Efficient XML Query Pattern Matching. Proc. ICDE: 141-152 (2002)

2. T. Böhme, E. Rahm: Supporting Efficient Streaming and Insertion of XML Data in RDBMS. Proc. 3rd DIWeb Workshop: 70-81 (2004)

3. N. Bruno, N. Koudas, D. Srivastava: Holistic twig joins: optimal XML pattern matching. Proc. SIGMOD: 310-321 (2002)

4. S.-Y. Chien, Z. Vagena, D. Zhang, V. J. Tsotras, C. Zaniolo: Efficient Structural Joins on Indexed XML Documents. Proc. VLDB: 263-274 (2002)

5. M. Dewey: Dewey Decimal Classification System. http://www.mtsu.edu/ vvesper/dewey.html

6. M. Fernandez, J. Hidders, P. Michiels, J. Simeon, R. Vercammen: Optimizing Sorting and Duplicate Elimination. Proc DEXA: 554-563 (2005).

7. M. Fontoura, V. Josifovski, E. Shekita, B. Yang: Optimizing Cursor Movement in Holistic Twig Joins, Proc. 14th CIKM: 784-791 (2005)

8. G. Gottlob, C. Koch, R. Pichler: Efficient algorithms for processing XPath queries. ACM Trans. Database Syst. 30(2): 444-491 (2005)

9. T. Härder, M. Haustein, C. Mathis, M. Wagner: Node Labeling Schemes for Dynamic XML Documents Reconsidered, accepted for Data \& Knowledge Engineering (2006)

10. Q. Li, B. Moon: Indexing and Querying XML Data for Regular Path Expressions. Proc. VLDB: 361-370 (2001)

11. Q. Li, B. Moon: Partition Based Path Join Algorithms for XML Data. Proc. DEXA: 160-170 (2003)

12. P. E. O’Neil, E. J. O’Neil, S. Pal, I. Cseri, G. Schaller, N. Westbury: ORDPATHs: InsertFriendly XML Node Labels. Proc. SIGMOD: 903-908 (2004)

13. I. Tatarinov et al.: Storing and Querying Ordered XML Using a Relational Database System. Proc. SIGMOD: 204-215 (2002)

14. Z. Vagena, M. M. Moro, V. J. Tsotras: Efficient Processing of XML Containment Queries using Partition-Based Schemes. Proc. IDEAS: 161-170 (2004)

15. Y. Wu, J. M. Patel, H. V. Jagadish: Structural Join Order Selection for XML Query Optimization. Proc. ICDE: 443-454 (2003).

16. C. Zhang, J. Naughton, D. DeWitt, Q. Luo, G. Lohmann, On Supporting Containment Queries in Relational Database Management Systems. Proc. SIGMOD: 425-436 (2001) 